\section{P125 A PRIMARY CARE AUDIT ON ASTHMA PATIENTS WITH FREQUENT EXACERBATIONS AND THE POTENTIAL IMPACT OF NATIONAL REVIEW OF ASTHMA DEATHS (NRAD) RECOMMENDATIONS}

${ }^{1} \mathrm{R}$ Chaudhuri, ${ }^{1} \mathrm{NC}$ Thomson, ${ }^{1} \mathrm{C}$ McCallum, ${ }^{2} \mathrm{H}$ O'Pray, ${ }^{3} \mathrm{ST}$ Barclay, ${ }^{1} \mathrm{D}$ Murray, ${ }^{4} \mathrm{~S}$ MacBride-Stewart, ${ }^{5} \mathrm{~V}$ Sharma, ${ }^{1} \mathrm{M}$ Shepherd, ${ }^{1} \mathrm{WT}$ Lee. ${ }^{1}$ Gartnavel General Hospital, Glasgow, UK; ${ }^{2}$ Contractor Services, NHS Greater Glasgow and Clyde, Glasgow, UK; ${ }^{3}$ Glasgow Royal Infirmary, Glasgow, UK; ${ }^{4}$ Prescribing and Pharmacy Support Unit, NHS Greater Glasgow and Clyde, Glasgow, UK; ${ }^{5}$ Queen Elizabeth University Hospital, Glasgow, $U K$

\subsection{6/thoraxjnl-2016-209333.268}

Background One of the key recommendations of $\mathrm{NRAD}^{1}$ is that patients who require 2 courses or more of oral corticosteroids (OCS) for asthma in the last 12 months should be referred to a specialist asthma service. This may put undue pressure on secondary care asthma services as the number of such patients is unknown.

Aims To identify asthma patients with recurrent exacerbations who would merit referral to secondary care, and to study the biopsychosocial factors associated with frequent exacerbations.

Methods Data were retrospectively collected on asthma patients aged $>18$ years from 10 primary care practices in the North Glasgow area over a 12-month period between 2014 and 2015. All prescriptions patients received in primary care were obtained. Only patients who received asthma treatment in the last 12 months were included. Patients were identified as a 'frequent exacerbator' if they had received $\geq 2$ courses of OCS for asthma, and a "short-acting beta 2-agonist (SABA) over-user" if they had required $>12$ SABA inhalers in the last 12 months.

Results Out of 2639 asthma patients studied, 7\% were frequent exacerbators, 5\% were SABA over-users, 1\% were both. Compared with all asthma patients, frequent exacerbators were older (mean age $58 \pm 16$ vs $48 \pm 17$ ) and more likely to be female (68\% vs 58\%). They had a higher prevalence of cigarette exposure and multiple co-morbidities including COPD, gastro-oesophageal reflux, anxiety, depression, rhinitis and osteoporosis. $41 \%$ of frequent exacerbators did not have an asthma review in primary care in the last 12 months and $42 \%$ had no previous input from secondary care. The total number of patients who would merit referral to a specialist asthma service from the 10 primary care practices was 78 over a 12 -month period.

Conclusions Frequent exacerbators and SABA over-users account for a small proportion of asthma patients attending primary care. The number of new referrals generated by the NRAD recommendation may put additional pressure on secondary care asthma services.

Funder supported by an educational grant from Novartis Pharmaceuticals.

\section{REFERENCE}

1 Levy ML. The national review of asthma deaths: what did we learn and what needs to change? Breathe (Sheff) 2015;11(1):14-24.

\section{\begin{tabular}{|l|l}
\hline P126 INSUFFICIENT ALLERGY DIAGNOSTICS IN SEVERE &
\end{tabular} ASTHMATIC PATIENTS IN GERMANY}

${ }^{1}$ IS Schreiber, ${ }^{2} \mathrm{CM}$ Mailaender. 'University Hospital, Otto-von-Guericke University, Magdeburg, Germany; ${ }^{2}$ Novartis Pharma GmbH, Nuremberg, Germany

\subsection{6/thoraxjnl-2016-209333.269}

Introduction Proper evaluation of the allergic sensitizations is inevitable in treating severe asthma. Besides seasonal allergens, perennial aeroallergens have to be considered. However, within the German reimbursement system testing of only 8 allergens per quarter is reimbursed by health insurances, and thus, analyses of relevant allergens beyond the 8 common ones is often not done.

Purpose The aim of this ongoing project is to gain data on sensitizations towards 35 perennial aeroallergens in severe asthmatic patients, in which no allergen could be detected in previous testing and who are thus considered non-atopic.

Methods 35 locally common perennial aeroallergens (mites, fungi, animal epithelia, insects) are tested via Immulite ${ }^{\circledR}$ (specific IgE in blood) in 600 severe asthmatic patients in Germany who had negative results in previous allergen testing by either Skin Prick Test or analyses of specific IgE. Furthermore, total IgE levels are determined and a general anamnesis is documented including historical allergen testing, comorbidities, symptoms, exposure to allergens etc.

Results In an interims analysis, $56.1 \%$ of 214 patients demonstrated at least one sensitisation towards a perennial aeroallergen despite they were considered non-atopic before. The most common sensitizations were found towards Rhizopus nigricans (16.8\%), Aspergillus fumigatus (15.9\%), Cat dander (11.1\%), Dermatophagoides farina (10.7\%) and Dog dander (9.9\%). $84.2 \%$ of patients were (partly) uncontrolled according to GINA classification of asthma control and $51.7 \%$ had $\geq 2$ exacerbations in the past 12 months. These results indicate a lack in diagnostics of perennial aeroallergens in severe asthmatic patients in Germany. The correct ascertainment of the allergic status is crucial to make optimal treatment decisions for the asthmatic patient.

\section{P127 BENEFITS AND SIDE EFFECTS OF NASAL IRRIGATION IN SEVERE ASTHMA}

A Clarke, A H Mansur. Birmingham Regional Severe Asthma Service, Heartlands Hospital, Heart of England NHS Foundation Trust, Birmingham, UK

\subsection{6/thoraxjnl-2016-209333.270}

Introduction and aim Rhinosinus disease is common in asthma and causes nasal congestion, post nasal drip and pain. This can lead to altered breathing patterns, particularly mouth breathing which exposes patients airways to cold dehumidified air and may aggravate their asthma. Nasal irrigation is accepted as an appropriate early intervention for managing allergic rhinitis but may be under-utilised in severe asthma. This study aims to evaluate the effects of nasal irrigation in a severe asthma population.

Method Thirty patients (23 females, mean age 47), with a confirmed diagnosis of severe asthma (22/30 73\% atopic) and rhinosinusitis took part in this study, none of the participants had previous nasal irrigation. Patients were taught to irrigate with the netti pot system $(0.9 \%$ saline), initially $1-2$ times each day and to titrate to individual needs. Symptoms were assessed with snott-22 questionnaires before and after three months of treatment, snott scores range from $0-110$, a difference of 8.9 points is the minimal change thought to be clinically significant. Data was also collected on ACQ scores, usage, reported benefits and side effects. Results 26 patients were using nasal irrigation at three month follow-up, mean initial snott scores were $63.2 \pm 22.9$ compared to $41.7 \pm 26.8$ post treatment $(\mathrm{p}=0.0001) .69 \%(18 / 26)$ patients showed clinically significant decreased nasal symptoms, with a mean improvement of 33.7 points. 19\% (5/26) showed no significant change and $12 \%(3 / 26)$ had a clinically significant increase in nasal symptoms. $88 \%(23 / 26)$ patients felt their nasal symptoms had improved, 62\% (16/26) felt their chest symptoms had 